\title{
UNIVERSITYOF
}

FORWARD

THINKING

WESTMINSTER用

WestminsterResearch

http://www.westminster.ac.uk/westminsterresearch

\section{Multi-Stage Complex Notch Filtering for Interference Detection and Mitigation to Improve the Acquisition Performance of GPS Arif, S., Coskun, A. and Kale, I.}

This is a copy of the author's accepted version of a paper subsequently published in the proceedings of the 14th Conference on PhD Research in Microelectronics and Electronics (PRIME 2018), Prague, Czech Republic, 02 to 05 Jul 2018.

The final published version is available online at:

https://doi.org/10.1109/PRIME.2018.8430324

(C) 2018 IEEE . Personal use of this material is permitted. Permission from IEEE must be obtained for all other uses, in any current or future media, including reprinting/republishing this material for advertising or promotional purposes, creating new collective works, for resale or redistribution to servers or lists, or reuse of any copyrighted component of this work in other works.

The WestminsterResearch online digital archive at the University of Westminster aims to make the research output of the University available to a wider audience. Copyright and Moral Rights remain with the authors and/or copyright owners.

Whilst further distribution of specific materials from within this archive is forbidden, you may freely distribute the URL of WestminsterResearch: ((http://westminsterresearch.wmin.ac.uk/)).

In case of abuse or copyright appearing without permission e-mail repository@westminster.ac.uk 


\title{
Multi-Stage Complex Notch Filtering for Interference Detection and Mitigation to Improve the Acquisition Performance of GPS
}

\author{
Syed Waqas Arif, Adem Coskun and Izzet Kale \\ Applied DSP and VLSI Research Group \\ Department of Engineering \\ University of Westminster \\ waqas.arif@my.westminster.ac.uk, a.coskun@westminster.ac.uk, kalei@westminster.ac.uk
}

\begin{abstract}
Continuous Wave Interferences (CWIs) can degrade the accuracy of a Global Positioning System (GPS) receiver and moreover it can completely deteriorate receiver's normal operation. In this paper a low-cost anti-jamming system design is presented for the mitigation and detection of CWIs for GPS receivers. The anti-jamming system comprises of parameterizable Complex Adaptive Notch Filter (CANF) module which is able to detect and excise single or multiple CWIs. The CANF module is composed of a first, second and third order infinite-impulse response filter with an Auto-Regressive Moving Averager structure. The proposed CANF detects the existence of the CWI and estimates JNR level of incoming signal by using the statistical value of the adaptive parameter $b_{0}$. The impact of the CANF module on the acquisition is analyzed. Moreover, a simple and innovative system level model is proposed which can utilize each CANF efficiently with threshold setting of JNR estimation within the adaptation block. Threshold setting parameters provide trade-off between effective excision of CWI, order of the filter and power consumption. This results in a parameterizable CANF module and provide effective solution for the mitigation of interferences with a high-power profile for GPS based applications.
\end{abstract}

Keywords-Anti-Jamming, Complex Notch Filter, GPS receivers, complex baseband processing

\section{INTRODUCTION}

Jamming and Anti-Jamming of the Global Navigation Satellite System (GNSS) have become a hot research topic over years due to the fast evolving GNSS technology and its rapid growth for the consumer based applications. Due to extremely low power of the GNSS signal received on the surface of the earth GNSS signal is prone to slightest of the RF interference directed to any GNSS receiver. Its effect on the received GNSS signal is unpredictable and severely degrade the quality of the signal making it impossible for the receiver to acquire and track it. It is essential to differentiate between types of interference either as wide or narrowband interference. An interference can be wide band for the civilian $\mathrm{C} / \mathrm{A}$ code, but if same interference is to be compared with long encrypt $\mathrm{P}(\mathrm{Y})$ code will be referred as a narrowband interference. Being wide or narrowband merely depend on the bandwidth of the GNSS signal. Both $\mathrm{C} / \mathrm{A}$ and $\mathrm{P}(\mathrm{Y})$ have respective signal bandwidth of $2.046 \mathrm{MHz}$ and $20.46 \mathrm{MHz}$ [1]. Intentional jamming is categorized as spoofing and jamming. Spoofing is transmission of a fake version of GNSS signal with more strength in order to deceive the receiver and hence
GNSS receiver will report incorrect position and timing data. Vast analysis of jammer signals have shown that any jamming signal can be modelled by linear frequency modulation which means jamming signal instantaneous frequency sweeps a range of frequencies in very small duration usually microseconds targeting the entire GNSS band to jam signal. The ideal GPS L1 signal at receiver front-end can be expressed as

$s(t)=\sqrt{2 P_{i}} D_{s}\left(t-\tau_{0}\right) C\left(t-\tau_{0}\right) \cos \left(2 \pi f_{L 1+D} t+\theta\right)$

where $P_{i}$ is the signal power, $D_{s}$ is the navigation data bit with chip rate of $50 \mathrm{~Hz}$, and $C$ is coarse C/A code running at chip rate of $1.023 \mathrm{MHz}$ and $\tau_{0}$ is the code phase delay. Frequency parameter $f_{L 1+D}$ represents the L1 carrier frequency with a Doppler shift of $f_{D}$ (where the sum of these two frequency components results in $f_{L 1+D}=1575.42 \mathrm{MHz} \pm f_{D}$ )[2]. It is assumed that sufficient bits are available from the ADC which provide enough dynamic range to accommodate interference signal without ADC saturation.

\section{JAMMING SIGNAL}

\section{A. Modelling Jamming Signal}

Jammers can radiate a variety of interferences signal. Main focus of this research is on Continuous Wave Interference (CWI) signals in the form of Complex Sinusoidal Wave Interference (CSWI) and Complex Chirp-Type Wave Interference (Single or Multi Saw-tooth function). These interferences are the most common type of interferences emitted by the commercially available jammers in the market.

\section{B. Jamming to Noise Ratio (JNR)}

In order to evaluate the performance of any anti-jamming algorithm, it is important to determine the Jamming to Noise ratio (JNR) and it is defined as

$J N R=10 \log _{10}\left(\frac{P_{i}}{P_{n}}\right)$

$P_{i}$ And $P_{n}$ are the respective powers of jamming signal and the noise [3]. Interference's power with respect to noise variance is defined by the JNR which can mathematically be represented by

$$
\frac{P_{i}}{P_{n}}=\frac{\frac{1}{2} A_{i}^{2}}{\sigma_{n}^{2}}=\frac{A_{i}^{2}}{F_{S} N_{0}}=\frac{A_{i}^{2}}{2 B_{I F} N_{0}}
$$




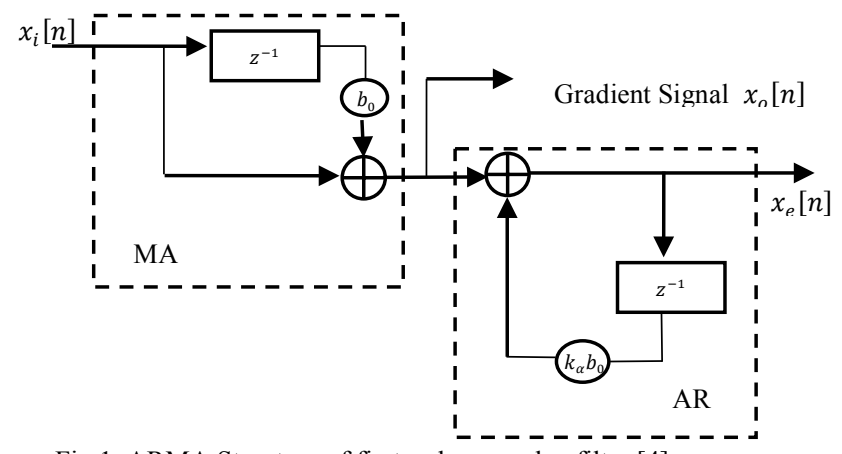

Fig 1. ARMA Structure of first order complex filter [4].

where $A_{i}$ is amplitude of narrowband CWI, $N_{0}$ is the noise per unit bandwidth, $F_{S}$ is the sampling frequency and $B_{I F}$ is the bandwidth of the front-end filter. The variance of the noise $\sigma_{n}^{2}$ is equal to $B_{I F} N_{0}$. This section presents modelling of the narrowband CWI through acquisition chain and its implications on the Cross Ambiguity Function (CAF) of the acquisition scheme. The fixed frequency complex sinusoidal CWI is present as an interference, it can be expressed by

$J_{\text {int }}(t)=\sqrt{P_{i}} \exp \left(j 2 \pi f_{i} T_{s}+\theta_{i}\right)$

where $P_{i}$ is the power of the narrowband CWI, $f_{i}$ is the frequency of the interferer and $\theta_{i}$ is the initial random phase of the interference. Hence GPS L1 signal at the RF front-end of the receiver is formulated by, $x_{i}(t)=s(t)+n(t)+J_{i n t}(t)$.

\section{HIGH ORDER COMPLEX NOTCH FILTER}

The structure of first order complex adaptive notch filter is shown in Fig.1, it comprise of two sections Moving Averaging (MA) and Autoregressive (AR). The z-domain transfer function of whole filter can be represented by (5) while $K=1$. Our observation on the excision of the CWI and complex sinusoidal signal using first order CNF are;

- $\quad$ The first order CNF filter under performs if the value of JNR is above $25 \mathrm{~dB}$ hence GPS L1 signal is unable to be acquired.

- The mitigation of high power CWI and complex sinusoidal signal requires notch filtering with deeper attenuation and tighter bandwidth.

These observations became motivation to undertake further analysis on higher order CNFs and incorporate them together such that, depending on the JNR power level system can decide which CNF order should be used for efficient excision of CWI interference without harming or distorting the actual signal

$H_{C N F}(z)=\prod_{K=1}^{K} \frac{1-b_{0} z^{-1}}{1-k_{\alpha} b_{0} z^{-1}}$

where the parameter $b_{0}$, is the complex coefficient of CNF and is also the zero location at the interference frequency and variable $k_{\alpha}$ determines the width of the notch. The Normalized Least Mean Square (NLMS) is used in this paper as it is less complex and suitable for real-time system for the practical

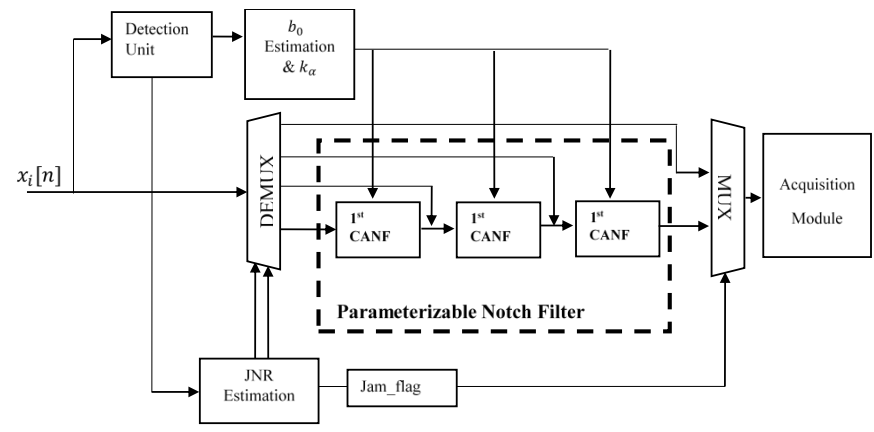

Fig 2. Proposed system level model.

integration into the GPS receivers. NLMS algorithm is used to minimize the cost function (6) and parameter $b_{0}$ is adapted by minimizing output of moving average part $x_{0}[n]$, given by the following equation [5].

$\operatorname{grad}(C[n])=\nabla_{z o}\left\{\left|x_{0}[n]\right|^{2}\right\}$

The $b_{0}$ coefficient of Complex Adaptive Notch Filter (CANF) can be updated using (7) where $\mu$ is the normalized step-size the value of which is very critical in determining the rate that the algorithm converge to a solution. A normalized step-size is used to avoid any misadjustment due to signal power level.

$b_{0}[n]=b_{0}[n-1]-\mu \times \operatorname{grad}(C[n])$

As given in [6], differentiation of cost function with respect to complex parameter $b_{0}$ in (6) leads to following equation:

$$
\nabla_{b_{0}}\left\{\left|x_{0}[n]\right|^{2}\right\}=-4 x_{0}[n]\left(x_{e}{ }^{*}[n-1]\right)
$$

Substituting (8) into (7) gives following adaptation equation for the coefficient $b_{0}$ of the CANF filter.

$b_{0}[n]=b_{0}[n-1]+\mu \times 4 x_{0}[n]\left(x_{e}{ }^{*}[n-1]\right)$

\section{PROPOSED SYSTEM LEVEL MODEL}

Proposed system level model is illustrated in Fig.2. Here the goal is to develop a paramterizable multi-stage notch filter that can switch from a lower to a higher order notch filter depending on the input JNR level. As the useful signal with interference enters the system the presence of the interference must be detected. Once interference is detected, system simultaneously estimate the zero location $b_{0}$ and JNR level. Depending on the level of estimated JNR, the signal $x_{i}[n]$ will be passed on to $1^{\text {st }}, 2^{\text {nd }}$ or $3^{\text {rd }}$ order CANF via a DEMUltipleXer (DEMUX) which is controlled by JNR estimation block in Fig.2. Jam_flag in Fig.2 determines whether incoming signal has to be filtered through CANF or pass directly to acquisition block when no interference is present. There is no point of using higher notch filter for low level of JNR which will consume more power. A simple and less power hungry first order notch filter can be used for mitigating interference with low JNR level. The working thresholds for $1^{\text {st }}, 2^{\text {nd }}$ and $3^{\text {rd }}$ order is estimated to be $26 \mathrm{~dB}, 46 \mathrm{~dB}$ and $51 \mathrm{~dB}$ respectively as marked with circles in Fig 3. The GPS L1 signal is known to be acquirable if output SNR at acquisition is $10 \mathrm{~dB}$ or above [7], as represented by the yellow dashed line in Fig.3. 


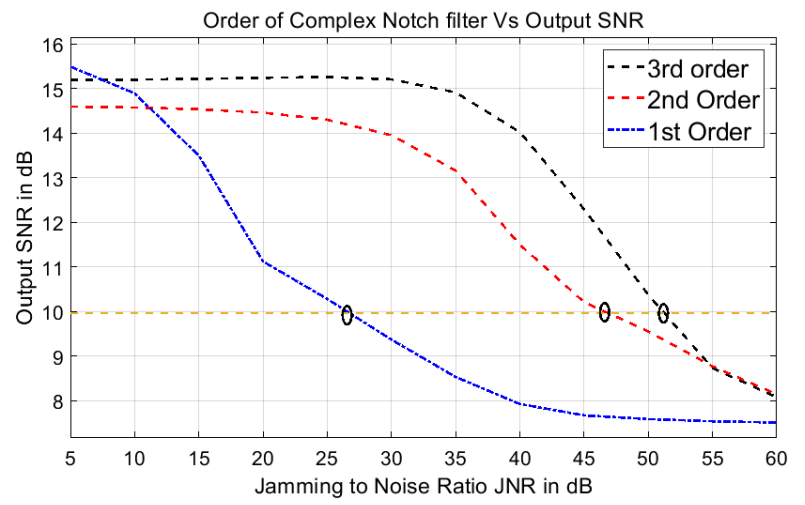

Fig 3. Output SNR vs. Order of Notch filter with same $b_{0}$ value for all the notch filter.

\section{PROPOSED JNR LEVEL ESTIMATION}

ALGORITHM: $J N R$ Level Estimation $\left(N_{p},\left|b_{0}\right|, \beta_{1}, \beta_{2}\right.$ and $\left.\beta_{3}\right)$

Collect $N_{p}$ number of samples of adaptive parameter $\left|b_{0}\right|$

Step 1: Compute mean of $\left|b_{0}\right|$ over $N_{p}$ number of samples

Step 2: Determine $\sigma_{\left|b_{0}\right|}^{2}$ the variance of $\left|b_{0}\right|$ using (10)

$\sigma_{\left|b_{0}\right|}^{2}=\frac{1}{N_{P}} \sum_{n=0}^{N_{P}-1} \operatorname{abs}\left(b_{0}[n]\right)^{2}-\operatorname{mean}\left(\left|b_{0}\right|\right)$

Now

$$
\begin{aligned}
& \text { If } \beta_{0}<\sigma_{\left|b_{0}\right|}^{2}<\beta_{1} \\
& \text { then DEMUX } X_{c t r l} 00 \text { ( No CANF filtering) } \\
& \text { elseif } \beta_{1}<\sigma_{\left|b_{0}\right|}^{2}<\beta_{2} \\
& \text { then DEMU } \left.X_{\text {ctrl }} 01 \text { ( } 1^{\text {st }} \text { Order }\right) \\
& \text { elseif } \beta_{2}<\sigma_{\left|b_{0}\right|}^{2}<\beta_{3} \\
& \text { elseif } \sigma_{\left|b_{0}\right|}^{2}>\beta_{3} \\
& \text { then DEMUX } X_{c t r l} 10\left(2^{\text {st }} \text { Order }\right) \\
& \text { then DEMU } \left.X_{c t r l} 11 \text { ( } 3^{\text {st }} \text { Order }\right)
\end{aligned}
$$

The $\beta_{1}, \beta_{2}$ and $\beta_{3}$ represent the threshold levels for the DEMUX to pass on the signal either to $1^{\text {st }}, 2^{\text {nd }}$ or $3^{\text {rd }}$ CANF. $\beta_{0}$ is the detection threshold for incoming interference. If mean of $\left|b_{0}\right|$ approximate to unity, it indicates the presence of CWI in the useful signal. The absolute value of $b_{0}$, i.e. $\left|b_{0}\right|$, fluctuates about its mean value whenever CWI is present in the useful signal and these fluctuation differ for different level JNR and hence the variance of $\left|b_{0}\right|$ can be used to estimate the power level of JNR. The range of fluctuation about unity strongly depend on the level of JNR. Fig.4illustrate the convergence curves of $\left|b_{0}\right|$ for different values of JNR. Different level of fluctuation of the magnitude of $\left|b_{0}\right|$ are more prominent in Fig.4 (a) which shows how $\left|b_{0}\right|$ eventually converges to unity for different levels of JNR but have different variance about unity. Another observation from Fig.4 (a) is, when JNR level is $0 \mathrm{~dB}$ (the pink curve), the modulus of parameter $b_{0}$ does try to converge to unity but the level of fluctuation is higher than any of the curve in Fig.4 (a). As the level of JNR increases the respective fluctuation for each successive JNR level damp down and decreases in amplitude. The simulation results in Fig.4 (b) gives an indication that rate of convergence of modulus of $b_{0}$ is different for different levels of JNR when rest of the parameters in simulation are kept unchanged.

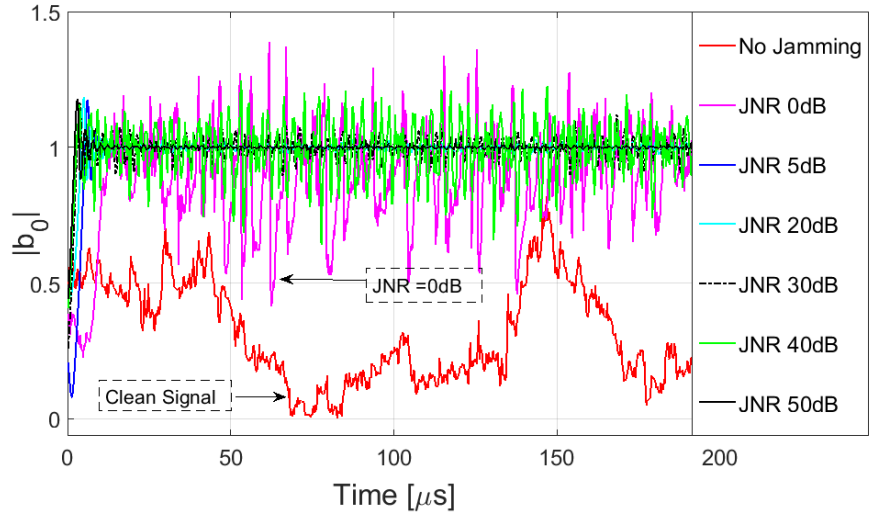

Fig 4. Convergence of magnitude of $\left|b_{0}\right|$ for different level of JNR. (a) shows behavior of $\left|b_{0}\right|$ from zero to $160 \mu$ s. (b) The zoomed in version of fig 4(a).

When interfering power is more, less number of iterations taken by NLMS algorithm to lock on the target frequency and the modulus of $b_{0}$ converges faster. Keeping the rest of the parameters the same or constant, it can be understood from the simulation results in this section that, the convergence of modulus of $b_{0}$ primarily depend on two factors, the power of narrowband CWI interference and the pole contraction parameter $k_{\alpha}$.As $\left|b_{0}\right|$ fluctuate about is mean value whenever $\mathrm{CWI}$ interference is present in the signal and these fluctuation differ for different level JNR, the variance of $\left|b_{0}\right|$ can be used to estimate the power level of JNR. Proposed interference power estimation is based on the variance of the modulus of $b_{0}$ about unity. Fig 5(b) shows how variance of modulus of parameter $b_{0}$ can be used as an estimate of power of jamming signal and JNR. The labels $\beta_{1}, \beta_{2}$ and $\beta_{3}$ in Fig. 5(b) represent three different threshold levels for $1^{\text {st }}, 2^{\text {nd }}$ and $3^{\text {rd }}$ CANF to be used in proposed system level model.

$\widetilde{J N} R_{\text {Level }}=f\left(\sigma_{\left|b_{0}\right|}^{2}\right)$

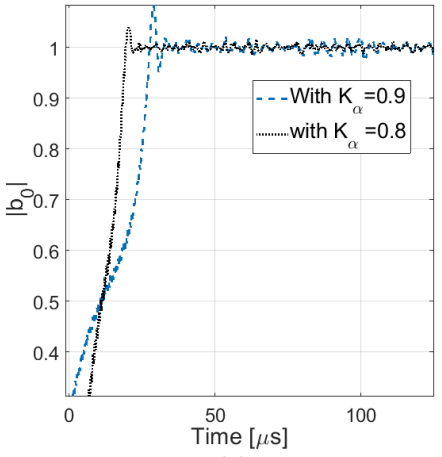

(a)

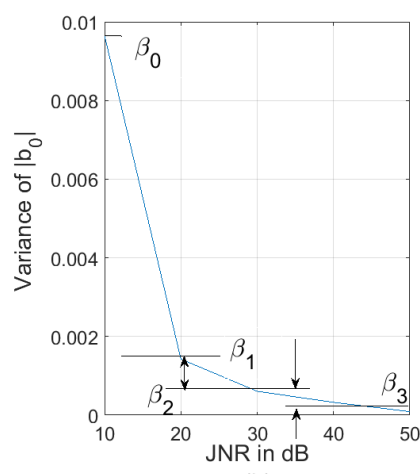

(b)
Fig 5. (a) Convergence of the $\left|b_{0}\right|$ of $1^{\text {st }}$ order notch filter for value of $k_{\alpha} 0.8$ and 0.9. (b) Setting up threshold to activate required CANF filter depending on variance of the magnitude of $b_{0}$

\section{SIMULATION RESULTS}

CANF is employed to remove and excise the CWI and complex chirp-type interference from GPS L1 signals. This section presents the simulation results for CWI in the form of time-frequency representation of signal before and after 
mitigation, 3D plot of the Cross Ambiguity Function (CAF) plot [8] at the output of the acquisition module after excision

(a)
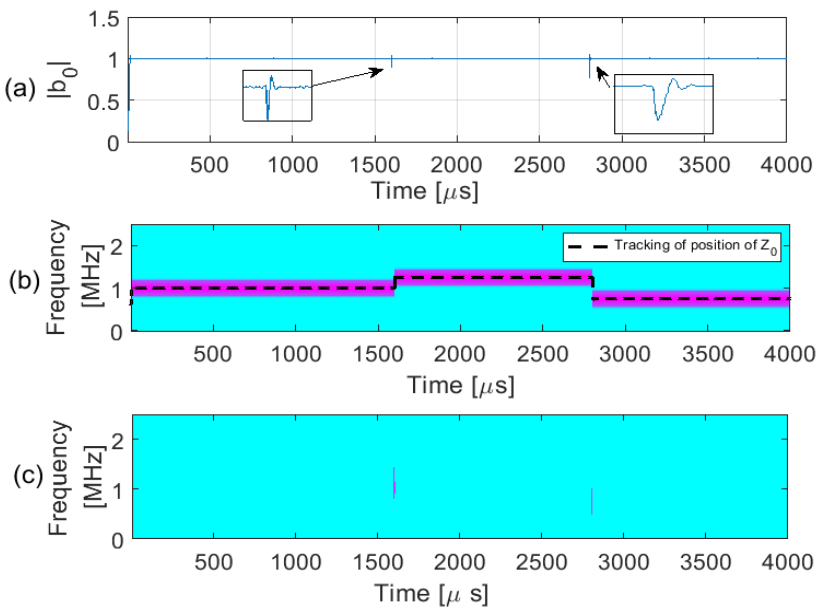

Fig 6. (a) Shows the convergence of $\left|b_{0}\right|$ over $4 \mathrm{~ms}$ length of data with CSWI at $0.75 \mathrm{MHz}, 1 \mathrm{MHZ}$ and $1.25 \mathrm{MHz}$ with $k_{a}=0.85$ and JNR level of $20 \mathrm{~dB}$. (b) Shows the time-frequency representation of GPS L1 signal with interference as indicated by thickline, and black dotted line represents the tracking position of $b_{0}$. (c) Shows the cleaned signal after mitigation of interference.

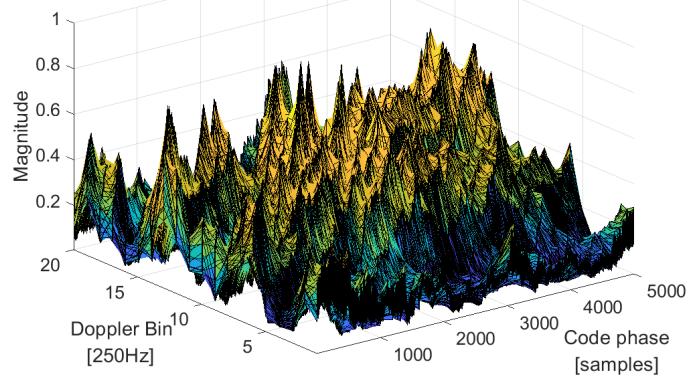

Fig 7. Evaluation of CAF at output of acquisition with interferences.
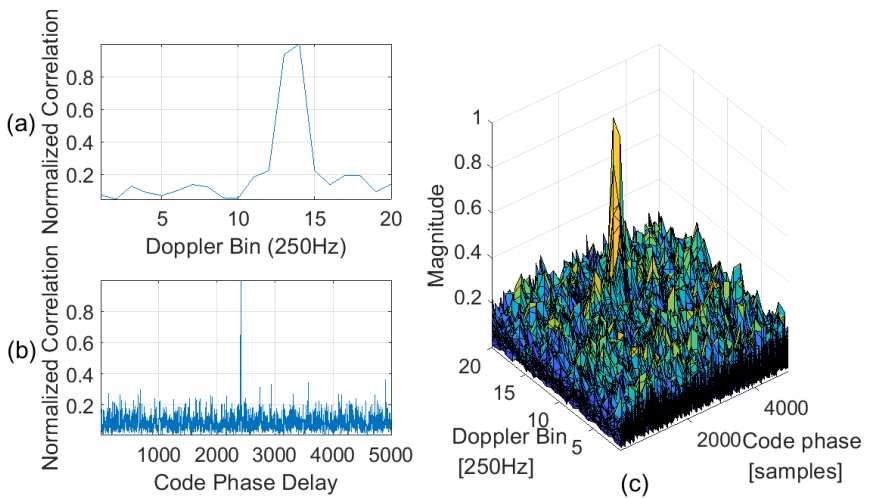

Fig 8. Output of acquisition block after mitigating CWI. (a) Doppler frequency estimation. (b) C/A code delay phase estimation. (c) CAF evaluation after mitigation of $\mathrm{CWI}$ at $0.75 \mathrm{MHz}, 1 \mathrm{MHZ}$ and $1.25 \mathrm{MHz}$. of the interference. Fig. 6(a) shows the convergence of adaptive parameter for first $4000 \mu s$ and the glitches in Fig. 6(a) shows the adaptation of $b_{0}$ to new interference frequency. Fig.6 (b) shows the time-frequency representation of CSWIs at $0.75 \mathrm{MHz}, 1 \mathrm{MHZ}$ and $1.25 \mathrm{MHz}$ and dotted brown line represents the CANF notch's center frequency following and adapting the interference precisely. The plot in Fig. 6(c) represent the cleaned signal after filtered through CANF. Fig. 8 and Fig.8 (c) shows evaluation of CAF before and after the mitigation of CSWIs at $0.75 \mathrm{MHz}, 1 \mathrm{MHZ}$ and $1.25 \mathrm{MHz}$. The correlation peak is in Fig.8 (c) prominent and visible as compare to multiple peaks in Fig.7, hence blindfold the receiver operations. Fig 8 (a) and (b) shows the estimated values of Doppler Shift and code phase delay respectively which are further use to determine the location.

\section{CONCLUSION}

A simple and innovative system level model is proposed utilizing multi-stage CANF efficiently with threshold setting of JNR estimation. Threshold setting parameters provide trade-off between effective excision of CWI and order of the filter. Hence power consumption and results in a parameterizable CANF which provide an effective solution for interference mitigation for GNSS based applications. Different aspects and properties of the parameter $b_{0}$ are exploited which could be beneficial for the estimation of JNR levels. Variance of the magnitude of parameter $b_{0}$ is proposed to set as threshold setting variable for estimation of level of JNR. It can be employed to toggle between different orders of the filter depending on level of JNR.

\section{REFERENCES}

[1] A. Ucar, E. Cetin, and I. Kale, "A low complexity DSP driven analog impairment mitigation scheme for Low-IF GNSS receivers," in 2008 IEEE/ION Position, Location and Navigation Symposium, 2008, pp. 865-870.

[2] E. D. Kaplan and C. Hegarty, Understanding GPS Principles and Applications. Boston: Boston : Artech House, 2006.

[3] M. Abdizadeh, J. T. Curran, and G. Lachapelle, 'New decision variables for GNSS acquisition in the presence of $\mathrm{CW}$ interference', IEEE Transactions on Aerospace and Electronic Systems, vol. 50, no. 4, pp. 2794-2806, Oct. 2014.

[4] H. Xiong, W. Zhang, Z. Du, B. He, and D. Yuan, 'Front-End Narrowband Interference Mitigation for DS-UWB Receiver', IEEE Transactions on Wireless Communications, vol. 12, no. 9, pp. 4328-4337, Sep. 2013.

[5] D. Borio, "Loop analysis of adaptive notch filters," IET Signal Processing, vol. 10, no. 6, pp. 659-669, 2016.

[6] S. S. Haykin, Adaptive Filter Theory. (3rd ed ed.) Englewood Cliffs, NJ ; London: Englewood Cliffs, NJ ; London : Prentice Hall, 1996.

[7] W. L. Mao, A. B. Chen, Y. F. Tseng, F. R. Chang, H. W. Tsao, and W. S. Huang, 'Design of Peak-finding Algorithm on Acquisition of Weak GPS Signals', in 2006 IEEE International Conference on Systems, Man and Cybernetics, 2006, vol. 3, pp. 1820-1825.

[8] D. Akopian, 'Fast FFT based GPS satellite acquisition methods', Sonar and Navigation IEE Proceedings - Radar, vol. 152, no. 4, pp. 277-286, Aug. 2005. 